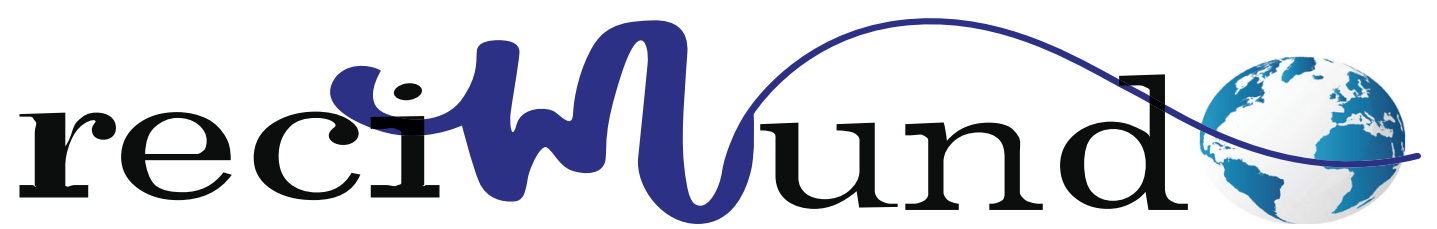

Revista Científica Mundo de la Investigación y el Conocimiento

DOI: 10.26820/recimundo/5.(4).dic.2021.356-371

URL: https://www.recimundo.com/index.php/es/article/view/1483

EDITORIAL: Saberes del Conocimiento

REVISTA: RECIMUNDO

ISSN: 2588-073X

TIPO DE INVESTIGACIÓN: Artículo de investigación

CÓDIGO UNESCO: 5909.01 Gestión Administrativa

PAGINAS: 356-371

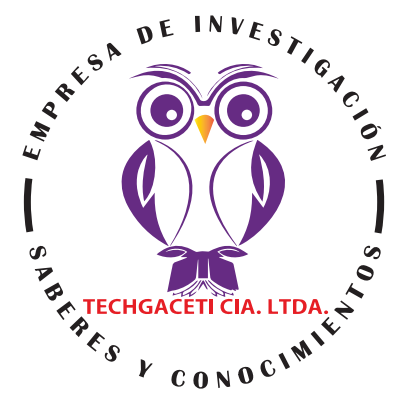

\title{
La gestión financiera como factor de la rentabilidad en las cooperativas de ahorro y crédito del Ecuador 2016-2020
}

Financial management as a factor of profitability in Ecuador savings and credit cooperatives 2016-2020

Gestão financeira como fator de rentabilidade nas cooperativas de poupanca e crédito do Equador 2016-2020

Dunia Maritza Yaguache Maza'; Julio Alberto Hennings Otoya²

RECIBIDO: 25/10/2021 ACEPTADO: 05/12/2021 PUBLICADO: 10/12/2021

1. Magister en Gerencia Contable y Financiera; Universidad Nacional de Loja, Loja, Ecuador; dunia.yaguache@ unl.edu.ec; (D) https://orcid.org/0000-0002-3058-3775

2. Doctor; Universidad Nacional Mayor de San Marcos, Lima, Perú; henningso@unmsm.edu.pe; (DD https://orcid. org/0000-0002-4195-5159

CORRESPONDENCIA

Dunia Maritza Yaguache Maza

dunia.yaguache@unl.edu.ec

Loja, Ecuador

๑ 


\section{RESUMEN}

La presente investigación, es de carácter no experimental, de diseño correlacional, siendo su alcance descriptiva, cualitativa, cuantitativa y aplicada. Su objetivo general es determinar si la gestión financiera influye en la rentabilidad del Sector Asociativo de las Cooperativas de Ahorro y Crédito de la Economía Popular y Solidaria del Ecuador 2016-2020. La recolección de información se realizó mediante la aplicación de una encuesta a representantes y/o funcionarios de las Cooperativas de Ahorro y Crédito del Segmento 1, así como la revisión de valores financieros de las Cooperativas de Ahorro y Crédito de segmento 1 del Ecuador, tomados de la página oficial de la Superintendencia de Economía Popular y Solidaria y el análisis documental realizado. Los resultados mostraron que existe correlación, la gestión financiera Sí influye en la rentabilidad de las Cooperativas de Ahorro y Crédito de la Economía Popular y Solidaria del Ecuador, 2016- 2020. Las hipótesis especificas mostraron que el desempeño financiero, SÍ incide en la rentabilidad de las Cooperativas de Ahorro y Crédito de la Economía Popular y Solidaria del Ecuador, en el periodo 2016-2020. Y la calidad del servicio, Sí incide en la rentabilidad de las Cooperativas de Ahorro y Crédito de la Economía Popular y Solidaria del Ecuador, en el periodo 2016- 2020. Además en términos de rentabilidad sobre patrimonio y sobre los activos el ROE y ROA, las Cooperativas de Ahorro y Crédito de la Economía Popular y Solidaria del Ecuador, 2016- 2020, segmento 1, presentan en los primeros períodos de estudio una tendencia creciente en ambos caso, 2016-2019, sin embargo, entre 2019 y 2020 se aprecia una disminución debido al contexto económico interno del país, en tanto que el año 2020, la crisis económica profundizada por la pandemia provocó que el sector cooperativo de ahorro y crédito del país no alcance los rendimientos del 2019, así presenta: ROE: $2016=0.07 ; 2017=0.07 ; 2018=0.10 ; 2019=0.08 ; 2020$ (marzo) =0.01; ROA: $2016=0.01 ; 2017=0.01$; $2018=0.01 ; 2019=0.01 ; 2020($ marzo $)=0.00$.

Palabras clave: Gestión, recursos, cooperativas, finanzas, rendimiento.

\section{ABSTRACT}

The present investigation, carried out is of a non-experimental nature, of correlational design, being its scope descriptive, qualitative, quantitative and applied. Its general objective is to determine if financial management influences the profitability of the Associative Sector of the Savings and Credit Cooperatives of the Popular and Solidarity Economy of Ecuador 20162020. The collection of information was carried out through the application of a survey to representatives and / or officials of the Savings and Credit Cooperatives of Segment 1, as well as the review of financial values of the Savings and Credit Cooperatives of segment 1 of Ecuador, taken of the official page of the Superintendency of Popular and Solidarity Economy and the documentary analysis carried out. The results showed that there is a correlation, financial management DOES influence the profitability of the Savings and Credit Cooperatives of the Popular and Solidarity Economy of Ecuador, 20162020. The specific hypotheses showed that the Savings and Credit Cooperatives of the Popular and Solidarity Economy of Ecuador that innovate, optimizing their financial management operational processes, YES, obtain better profitability in the results of the 2016-2020 period. Financial performance DOES affect the profitability of the Savings and Credit Cooperatives of the Popular and Solidarity Economy of Ecuador, in the period 2016-2020. And the quality of the service, DOES affect the profitability of the Savings and Credit Cooperatives of the Popular and Solidarity Economy of Ecuador, in the period 20162020. In addition, in terms of profitability on equity and on assets, ROE and ROA, the Savings and Credit Cooperatives of the Popular and Solidarity Economy of Ecuador, 2016-2020, segment 1, present in the first study periods a growing trend in both In this case, 2016-2019, however, between 2019 and 2020 there is a decrease due to the internal economic context of the country, while in 2020, the economic crisis deepened by the pandemic caused the country's savings and credit cooperative sector does not reach the returns of 2019, this is how it presents: ROE: $2016=0.07 ; 2017=0.07 ; 2018=0.10 ; 2019$ $=0.08 ; 2020($ March $)=0.01 ;$ ROA: $2016=0.01 ; 2017=0.01 ; 2018=0.01 ; 2019=0.01 ; 2020($ March $)=0.00$.

Keywords: Management, resources, cooperatives, finance, performance.

\section{RESUMO}

A presente investigação, realizada é de natureza não experimental, de desenho correlacional, sendo seu escopo descritivo, qualitativo, quantitativo e aplicado. Seu objetivo geral é determinar se a gestão financeira influencia a rentabilidade do Setor Associativo das Cooperativas de Poupança e Crédito da Economia Popular e Solidária do Equador 2016-2020. A coleta de informações foi realizada por meio da aplicação de uma pesquisa a representantes e/ou funcionários das Cooperativas de Poupança e Crédito do Segmento 1, bem como a revisão dos valores financeiros das Cooperativas de Poupança e Crédito do segmento 1 do Equador, tomadas da página oficial da Superintendência de Economia Popular e Solidária e a análise documental realizada. Os resultados mostraram que há uma correlação, a gestão financeira influencia a rentabilidade das Cooperativas de Poupança e Crédito da Economia Popular e Solidária do Equador, $2016-2020$. As hipóteses específicas mostraram que as Cooperativas de Poupança e Crédito da Economia Popular e Solidária do Equador que inovam, otimizando seus processos operacionais de gestão financeira, SIM, obtêm melhor rentabilidade nos resultados do período 2016-2020. O desempenho financeiro Afeta a rentabilidade das Cooperativas de Poupança e Crédito da Economia Popular e Solidária do Equador, no período 2016-2020. E a qualidade do serviço Afeta a rentabilidade das Cooperativas de Poupança e Crédito da Economia Popular e Solidária do Equador, no período 2016-2020. Além disso, em termos de rentabilidade sobre o patrimônio e sobre os ativos, ROE e ROA, as Cooperativas de Poupança e Crédito da Economia Popular e Solidária do Equador, 2016-2020, segmento 1, apresentam nos primeiros períodos de estudo uma tendência crescente tanto em neste caso, 2016-2019, no entanto, entre 2019 e 2020 há um decréscimo devido ao contexto económico interno do país, enquanto em 2020, a crise económica aprofundada pela pandemia fez com que o setor cooperativo de poupança e crédito do país não chegasse ao retornos de 2019, assim apresenta: ROE: 2016 = 0,07; $2017=0,07 ; 2018=0,10 ; 2019=0,08 ; 2020($ março $)=0,01 ; \operatorname{ROA}: 2016=0,01 ; 2017=0,01 ; 2018=0,01 ; 2019=0,01$; $2020($ março $)=0,00$.

Palavras-chave: Gestão, recursos, cooperativas, finanças, desempenho. 


\section{Introducción}

La economía social y solidaria, en las últimas décadas, ha tomado incidencia en algunos países, así en la Constitución de la República del Ecuador, establece que el régimen de desarrollo tendrá como uno de los objetivos: Construir un sistema económico, justo, democrático, productivo, solidario y sostenible basado en la distribución igualitaria de los beneficios del desarrollo, de los medios de producción y en la generación de trabajo digno y estable, por lo que se consideró pertinente, investigar las Organizaciones del Sector Cooperativo, que según la Ley de Economía Popular y Solidaria son entendidas como sociedades de personas que se han unido en forma voluntaria para satisfacer sus necesidades económicas, sociales y culturales en común, mediante una empresa de propiedad conjunta y de gestión democrática, con personalidad jurídica de derecho privado e interés social. El cooperativismo, después de enfrentar un siglo de grandes obstáculos, promovió de forma destacada, el acceso de amplios sectores sociales a diversas formas asociativas de producción, sobre todo en el campo del ahorro y crédito y del transporte, desarrolló un servicio de crédito, basado en el conocimiento y confianza de los socios cooperados y en la auto exigencia de brindar un servicio accesible a la población de bajos ingresos. Por lo que se inició la investigación, sobre: La Gestión Financiera como factor de competitividad en la rentabilidad financiera del Sector Asociativo de las Cooperativas de Ahorro y Crédito de la Economía Popular y Solidaria del Ecuador 2016-2020.

El autor Guerrero (2003), menciona que los "Los retos de la gestión financiera frente a la planeación estratégica de las organizaciones y la globalización". El autor Barillas (2012) manifiesta que el surgimiento de China y otras economías asiáticas plantea desafíos particulares para las economías de América Latina, muchas de las cuales son ricas en materias primas y recursos naturales que sus nuevos socios necesitan. Por su parte, los inversionistas asiáticos con mucha liquidez están mirando a América Latina como una gran oportunidad de negocio, comprando acciones en la industria manufacturera, de construcción y otras industrias en algunos de los mercados de mayor crecimiento de la región. Esto está transformando la relación entre ambas regiones.

La Constitución (2008) estableció, que nuestro sistema económico es social y solidario y está integrado por tres subsistemas, el privado, el público y el popular y solidario, de lo que se destaca que el sistema popular y solidario, debe propender a fortalecer el trabajo asociativo y mejorar la producción para apuntalar la economía de los pequeños empresarios.

Según la Red Financiera Rural (2014) manifiesta que una vez que ha sido aprobado el Código Orgánico Monetario y Financiero, se contemplan varios aspectos interesantes en relación a las micro finanzas en el país. Entre los aspectos a destacar tenemos que la Junta de Regulación Monetaria y Financiera tiene amplios poderes para la emisión de normativa en tiempos cortos. Se integra a las instituciones financieras populares y solidarias, bajo un marco legal común derogando varios artículos de la Ley Orgánica de la Economía Popular y Solidaria en relación a la supervisión del sector. Constituyéndose así en un marco legal que protege los recursos invertidos en las microfinanzas en el país, lo que brinda confianza a los socios e inversionistas.

En Ecuador las instituciones financieras están obligadas a publicar entre otros indicadores, los de eficiencia por lo menos una vez al año con el fin de poner a disposición de accionistas, usuarios y público en general información económico-financiera que dictamine y refleje el desempeño de cada una de sus actividades, lo que consta en el Artículo 221 del Código Orgánico Monetario y Financiero. 


\section{LA GESTIÓN FINANCIERA COMO FACTOR DE LA RENTABILIDAD EN LAS COOPERATIVAS DE AHORRO Y CRÉDITO DEL ECUADOR 2016-2020}

Sánchez D. M (2015) La administración imparte efectividad a los esfuerzos humanos, ayuda a optimizar el manejo y selección del personal, equipo, materiales, dinero y relaciones humanas. Se mantiene al frente de las condiciones cambiantes y proporciona previsión y creatividad. El mejoramiento es su consigna constante.

La administración se aplica en todo tipo de corporaciones: 1) la utilizan todos los administradores en todos los niveles de una corporación, 2) es la meta de todos los administradores en todos los niveles de la corporación 3) la administración se ocupa del rendimiento esto implica eficacia y eficiencia.

Los elementos de la administración son: Personas o recursos humanos, Actividades, Objetivos, Recursos financieros, equipos, maquinarias, etc. Métodos: conocimientos, aplicaciones tecnológicas y operativas.

\section{Gestión Financiera}

Desarrollo Empresarial: Para el autor Delfín \& Acosta (2016), el desarrollo empresarial articula diferentes elementos con los que el empresario puede llevar a una organización hacia el logro de sus objetivos. Siendo así necesario considerar elementos como: crecimiento económico, cultura empresarial, liderazgo, gestión del conocimiento e innovación. Es un concepto integrador con el que se puede lograr un impacto positivo en las organizaciones mediante el reconocimiento de las capacidades del capital humano. Alcanzar un desarrollo empresarial le permitirá al empresario de una Pyme aprovechar las oportunidades que se le presentan a la empresa en un entorno globalizado y articular su desarrollo sostenible.

Crecimiento Económico: El crecimiento en una empresa se establece con referencia a su mayor o menor productividad, y se entiende a la productividad como la habilidad o facultad de producir, lo que lleva implícito el reconocimiento del esta-do y la manera como fueron utilizados los diversos insumos en el proceso productivo. En este sentido, la productividad condensa el problema central de la economía: hacer un mejor y mayor uso de los recursos disponibles. Así pues, todo sistema económico tendría como objetivo obtener una mayor productividad (Pozos \& Márquez, 2016, pág. 187).

EL autor Córdoba (2013) precisa que la gestión financiera, es una disciplina que se ocupa de determinar el valor y tomar decisiones, por consiguiente, se interesa en la adquisición, financiamiento, y administración de activos, siendo una meta global, y que por ende se convierte a la misión y visión en operaciones monetarias. Y consecutivamente la gestión financiera es el proceso que involucra el uso racional y responsable de todos los ingresos y egresos en todas las estructuras organizacionales.

Según el autor Burk \& Lehnman (2004), señalan que la gestión financiera para las pequeñas y medianas empresas, puede resultar de interés para las mismas, dado que les pueden permitir permanecer y madurar dentro del tejido empresarial y escalar su crecimiento.

Las Posibles Metas Financieras: "Las posibles metas financieras, se podrían encontrar algunas ideas como las siguientes: Sobrevivir, evitar las dificultades financieras y la quiebra, derrotar a la competencia, maximizar las ventas o la participación de mercado, minimizar los costos, maximizar las utilidades, mantener un crecimiento constante de las ganancias según (Jordan, 2006).

La Gestión Financiera a Largo Plazo: La gestión financiera a largo plazo se ocupa de la planificación futura de la empresa, buscando el modo de efectuar las inversiones en activos que generen flujos de efectivo constantes, controlando la rentabilidad generada por las mismas, así como focalizando la capacidad de endeudamiento de la empresa. 
La Gestión Financiera a Corto Plazo: La gestión financiera a corto plazo, se ocupa de la financiación del activo corriente. Es decir, financiar las inversiones a corto plazo como inventarios y otros.

La gestión financiera mantiene un protagonismo en la empresa y por eso se analiza cada una de sus variables: financiamiento, endeudamiento, rentabilidad, crecimiento, utilización de la información contable y financiera (Bojórquez, Perez, \& Gonzalez, 2013).

La Gestión Financiera Integral: La Gestión Financiera Integral. es el proceso mediante el cual las organizaciones son direccionadas al cumplimiento de sus objetivos, resaltando la importancia de las variables constitutivas de los procesos financieros empresariales, pero con un enfoque integrador de los demás componentes que hacen parte de la gestión organizacional, tales como: las estructuras administrativas, de procesos, el sistema productivo, los recursos humanos y los sistemas de calidad, entre otros (Correa, Ramírez, \& Castaño, 2009).

\section{Desempeño Financiero:}

Una de las acciones más importantes dentro de la administración de los recursos económicos, pero que es poco usual llevarla a cabo, tanto en personas como en pymes, es la de evaluar la gestión financiera. (Ortìz Gonzàlez, 2015).

Según el autor Nevado (2006), manifiesta que el funcionamiento de cualquier empresa requiere un conjunto organizado de bienes, que, valorado en términos monetarios, constituye su inversión o activo según la terminología contable. Sus componentes pueden ser materiales, inmateriales, derechos de cobro ante terceros, monetarios o financieros. Todos ellos tienen como atributo común que contribuyen, directa o indirectamente a obtener una renta o ingreso futuro.

\section{Rentabilidad}

La medición del proceso de creación de valor económico en la empresa debe enfocarse en toda una organización. Descender en cascada a todas y cada una de las unidades de negocio, para precisar en dónde se genera valor y en dónde la capacidad para hacerlo requiere de una estrategia para alinear recursos y potenciales. Es necesario entender que el valor económico no está cifrado sólo en los aspectos financieros, sino en todos los componentes que interactúan para que una empresa sea rentable, pero también una fuente productiva que considere la participación en el mercado, la satisfacción del cliente, la rotación de personal y la actualización de los cuadros de mando, entre otros. También hay que tener claro que la implementación de un sistema para medir la creación de valor es más que una técnica: es una forma de ver las cosas de manera diferente. En el fondo es una filosofía gerencial para administrar empresas (Franklin, 2007).

Al referirse a la empresa desde su forma operativa, Nevado (2006), citado por Pozos \& Márquez (2016) menciona que los objetivos fundamentales, en lo que hace referencia a la gestión, control y análisis de las actividades económicas y financieras, podrían englobarse en los siguientes:

1. Estabilidad económica, rentabilidad-seguridad. Se consigue con una adecuada proporcionalidad entre el activo fijo y circulante que permita una rentabilidad máxima con un cierto grado de seguridad financiera. La permanencia de la empresa de forma indefinida es algo a lo que ningún agente económico ni social quiere renunciar por lo que se pretende la máxima rentabilidad, pero sin poner en peligro su continuidad en el tiempo, por ser este el objetivo básico. Por otra parte, precisa proveerse de fon-dos financieros propios o ajenos, a corto y a largo plazo. De la combinación de los 


\section{LA GESTIÓN FINANCIERA COMO FACTOR DE LA RENTABILIDAD EN LAS COOPERATIVAS DE AHORRO Y CRÉDITO DEL ECUADOR 2016-2020}

mismos va a depender tanto su influencia en la rentabilidad de los recursos propios como en su estabilidad y viabilidad futuras.

2. Estabilidad financiera, solvencia-estabilidad. Se consigue con la concreción de las tres condiciones básicas: contar con los recursos financieros precisos, disponer de los mismos en el momento adecuado y oportuno, así como durante el tiempo previsto y al mínimo coste posible. Para la empresa la combinación optima de las tres condiciones posibles supone asegurar liquidez y considerar el nivel de solvencia ante los acreedores, lo que implica a su vez, la determinación de la estructura que le permita obtener la rentabilidad máxima de los recursos propios y su estabilidad necesaria para mantener es posición en el tiempo (págs. 189-190).

Según el autor Córdoba (2013), la rentabilidad es una relación porcentual que nos indica cuanto se obtiene a través del tiempo por cada unidad de recurso invertido, es la relación entre los ingresos y los costos.

Rentabilidad económica: relacionada con los negocios y corresponde al rendimiento operativo de la empresa, se mide por la relación entre la utilidad operativa, antes de intereses e impuestos, y el activo o la inversión operativa.

Rentabilidad financiera: es la rentabilidad del negocio desde el punto de vista del accionista, o sea cuanto se obtiene sobre el capital propio después de descontar el pago de la carga financiera.

Rentabilidad total: es la rentabilidad medida en términos de la relación entre la utilidad neta y el capital total.

Rendimiento sobre el patrimonio (ROE). - Mide el rendimiento ganado sobre la inversión de los accionistas comunes en la empresa. (SEPS, 2017). Mide el nivel de re- torno generado por el patrimonio invertido por los accionistas o socios de la entidad financiera.

\section{FÓRMULA DE CÁLCULO}

ROE $=\frac{\text { Utilidad Neta }}{\text { Patrimonio }}$

Interpretación del indicador. - Mientras menor sea el indicador, la entidad muestra que no tendría los suficientes recursos para cubrir la remuneración o beneficios a sus accionistas o socios.

Rendimiento sobre el Activo ROA. - Mide el nivel de retorno generado por el activo. Es una medida de eficacia en el manejo de los recursos de la entidad.

\section{FÓRMULA DE CÁLCULO}

ROA $=\frac{\text { Utilidad Neta }}{\text { Activo }}$

Interpretación del indicador. - Mientras menor sea el indicador, la entidad muestra que no podrá generar los suficientes ingresos que permitan fortalecer el patrimonio, Así como muestra el rendimiento del activo.

\section{Utilidad Bruta en Operaciones}

La palabra utilidad la entendemos como el interés o provecho que se obtiene de algo. Podemos hacer una primera distinción entre:

Utilidad total, se trata de la utilidad que brinda la cantidad consumida de un bien. Utilidad marginal, que se refiere al incremento que se produce en la utilidad total al consumir la última unidad de dicho bien. En economía y finanzas, la palabra utilidad se asocia a la ganancia que obtenemos a raíz de un bien o una inversión. 
La Utilidad bruta como la diferencia entre los ingresos de una empresa y los costos de ventas. Es importante saber que la Utilidad bruta no toma en cuenta los costos fijos (los costos laborales del personal, costos legales y costos de comercialización y alquiler), los costos financieros como son los impuestos o los pagos de intereses sobre deuda. Cálculo:

\section{FÓRMULA DE CÁLCULO}

\section{Utilidas Bruta en Operaciones}

$=$ Ingresos - Costos y Gastos

\section{Métodos o metodología}

La investigación es no experimental cuantitativa. Se realizó con información que se obtuvo de las Organizaciones de Economía Popular y Solidaria del cantón Loja. Se utilizó una encuesta de tipo Likert, con base a los datos obtenidos de la Superintendencia de Economía Popular y Solidaria. A su vez es una investigación que tiene un diseño de tipo cualitativo, cuantitativo y aplicada porque se enfrentó el problema proponiendo alternativas de solución. El nivel de la investigación es Aplicada dado a que está Orientada a lograr un nuevo conocimiento destinado a procurar soluciones de problemas prácticos.

La población es de 508 Cooperativas de Ahorro y Crédito de la Economía Popular y Solidaria del Ecuador de los segmentos del 1 al 5. La población está constituida por dos grupos definidos, como los Representantes Legales de las Cooperativas y los administradores. Muestra de conveniencia de Segmento 1, igual a 31 Cooperativas de Ahorro y Crédito de la Economía Popular y Solidaria del Ecuador. Encuestas a Representantes Legales, Administradores y Socios de las Cooperativas, se realizó la revisión de valores Financieros de las Cooperativas de Ahorro y Crédito de segmento 1 de la Economía Popular y Solidaria del Ecuador.
Se recopiló información bibliográfica que permita sustentar las teorías de la Gestión financiera y la competitividad de las organizaciones de economía popular y solidaria del Cantón Loja, información que fue organizada en base a los objetivos planteados para la verificación de hipótesis.

\section{Resultados y discusión}

Sector Cooperativo, Sistema Económico en Ecuador, antecedentes y generalidades.

En la actualidad, la economía popular y solidaria en el país concentra a más de 5 millones de asociados, de los cuales, el $28.03 \%$ son mujeres vinculadas al sector y el $21.75 \%$ es población joven. En cuanto a los activos que posee el sector financiero popular y solidario estos rebasan los $16 \mathrm{mil}$ millones de dólares, que equivalen al 15\% del PIB en Ecuador. De ellas, el 32\% de las organizaciones de la EPS pertenecen al sector agropecuario, uno de los mayores generadores de empleo en el país, pues cuenta con el $29.4 \%$ de los trabajadores vinculados. La primera Ley de Cooperativas se registra en Ecuador como intervención del Estado en 1937. Aquí se menciona a la Caja de Ahorro y Cooperativa de Préstamos de la Federación Obrera de Chimborazo como la primera organización financiera en 1927. En su análisis se hace un registro de cómo el número de instituciones de ahorro y crédito evolucionan, junto a datos de la Superintendencia de Economía Popular y Solidaria con el fin de contar con una visión actual de la situación cooperativa financiera del país (Luque González \& Peñaherrera Melo, 2021).

Desde 2018, las cooperativas son clasificadas según los montos en activos. En el caso del segmento 1, son 30 de acuerdo a las publicaciones de balances mensuales que realiza la Superintendencia de Economía Popular y Solidaria. De éstas, 5 son entidades indígenas de las cuales 3 se ubican en la provincia de Tungurahua. Las COAC indí- 


\section{LA GESTIÓN FINANCIERA COMO FACTOR DE LA RENTABILIDAD EN LAS COOPERATIVAS DE AHORRO Y CRÉDITO DEL ECUADOR 2016-2020}

genas representan el 7,8\% de los activos del total del segmento. En la provincia de Tungurahua se cuenta además con Oscus, San Francisco, El Sagrario y Cámara de Comercio de Ambato; por tanto, el monto total en activos representa el 15,97 \% del sistema cooperativo del segmento 1 en Ecuador, ocupando el tercer lugar después de Azuay y Pichincha. Entre las cooperativas, destaca Juventud Ecuatoriana Progresista Ltda. - JEP, que representa más del 20\% del segmento en Ecuador y Jardín Azuayo cuya participación en el mercado es de 9,04\%. Ambas cooperativas tienen su matriz en la ciudad de Cuenca (zona Austral), con presencia nacional a través de agencias y cajeros (ATM) (Luque González \& Peñaherrera Melo, 2021).

Tabla 1. Clasificación por segmentos de las Cooperativas de Ahorro y Crédito en Ecuador.

\begin{tabular}{lll}
\hline Segmento & \multicolumn{1}{c}{ COAC } & \multicolumn{1}{c}{ Activos (USD) } \\
\hline 1 & 33 & Mayor a $80^{\prime} 000.000,00$ \\
2 & 42 & Mayor a $20^{\prime} 000.000,00$ hasta $80^{\prime} 000.000,00$ \\
3 & 80 & Mayor a 5'000.000,00 hasta 20'000.000,00 \\
4 & 161 & Mayor a 1'000.000,00 hasta 5'000.000,00 \\
5 & 215 & Hasta 1'000.000,00 \\
\hline
\end{tabular}

Fuente: (Luque González \& Peñaherrera Melo, 2021)

Tabla 2. Rubros financieros de las Cooperativas de Ahorro y Crédito del Sistema financiero Popular y Solidario (SFPS). Segmento 1.

\begin{tabular}{llllll}
\hline & \multicolumn{2}{l}{ Total, Segmento 1} & & & \\
\cline { 2 - 6 } Descripción & $31 / 12 / 2016$ & $31 / 12 / 2017$ & $31 / 12 / 2018$ & $31 / 12 / 2019$ & $30 / 3 / 2020$ \\
ACTIVO & $6,641,610,269.30$ & $8,293,156,274.24$ & $9,698,176,276.79$ & $11,670,686,532.96$ & $11,852,142,054.15$ \\
PASIVOS & $5,664,516,759.66$ & $6,917,521,924.05$ & $8,305,817,185.95$ & $10,027,085,223.70$ & $10,169,695,746.84$ \\
PATRIMONIO & $977,093,509.64$ & $1,375,634,350.19$ & $1,392,359,090.84$ & $1,643,601,309.26$ & $1,658,408,452.90$ \\
GASTOS & $739,456,748.81$ & $814,049,754.53$ & $1,047,987,198.20$ & $1,312,722,944.70$ & $365,775,866.92$ \\
INGRESOS & $803,291,532.23$ & $908,511,506.54$ & $1,181,456,522.82$ & $1,451,179,373.01$ & $389,813,721.33$ \\
RESULTADOS & $63,834,783.42$ & $94,461,752.01$ & $133,469,324.62$ & $138,456,428.31$ & $24,037,854.41$ \\
\hline
\end{tabular}

Fuente: Los Autores 
Tabla 3. Puntos de atención del Sector financiero Popular y Solidario por segmentos.

\begin{tabular}{|c|c|c|c|c|c|c|}
\multicolumn{5}{|c|}{ PUNTOS DE ATENCIÓN DEL SECTOR FINANCIERO POPULAR Y SOLIDARIC } \\
\hline Segmento & Matriz & Agencia & Sucursal & $\begin{array}{c}\text { Cajero } \\
\text { automático }\end{array}$ & Otros** & TOTAL \\
\hline Segmento 1* & 40 & 576 & 65 & 1250 & 479 & 2410 \\
\hline Segmento 2 & 46 & 217 & 53 & 50 & 31 & 397 \\
\hline Segmento 3 & 84 & 147 & 41 & 6 & 37 & 315 \\
\hline Segmento 4 & 164 & 118 & 16 & 2 & 24 & 324 \\
\hline Segmento 5 & 191 & 21 & 4 & 1 & 9 & 226 \\
\hline TOTAL & 525 & 1079 & 179 & 1309 & 580 & 3672 \\
\hline
\end{tabular}

Fuente: Los Autores

\section{Análisis, Interpretación y Discusión de Resultados}

\section{Gestión Financiera}

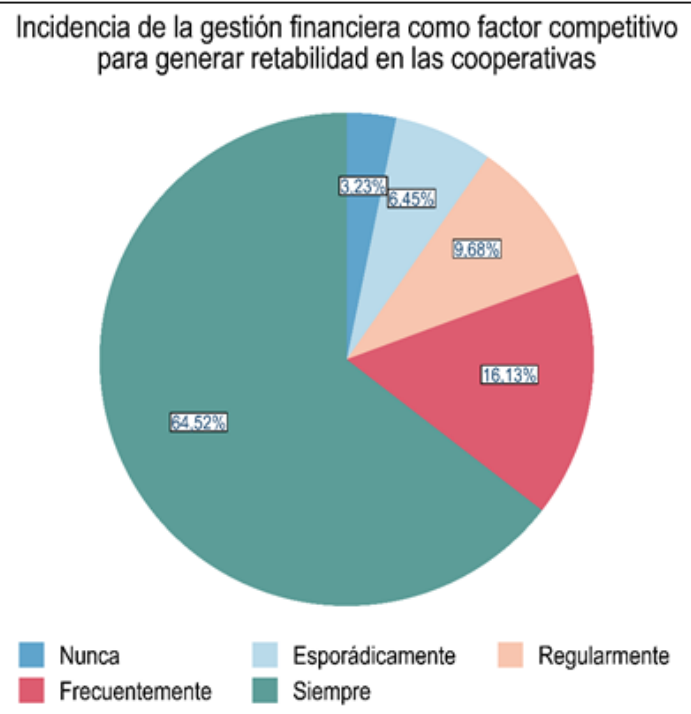

Figura 1. Incidencia gestión financiera como factor rentabilidad.

Fuente: Los Autores

En cuanto a la gestión financiera se tiene que, si se considera a ésta como un factor competitivo para generar rentabilidad en las Cooperativas, se reporta que más del $60 \%(64,52 \%)$ de los registros encuestados reportan que Siempre la entidad considera este elemento como un factor asociado a la rentabilidad. Dicho porcentaje es equivalente a 20 entidades. Un 16,13\% de estas entidades, siendo estas 5, indican que dicha consideración se da frecuentemente; un $9,68 \%$ de entidades, las que ascienden a 3 , indican que solo se da regularmente; $6,45 \%$ ( 2 entidades) y $3,23 \%$ ( 1 entidad) reportan que este hecho solo se registra esporádicamente o nunca, respectivamente a cada monto en mención.

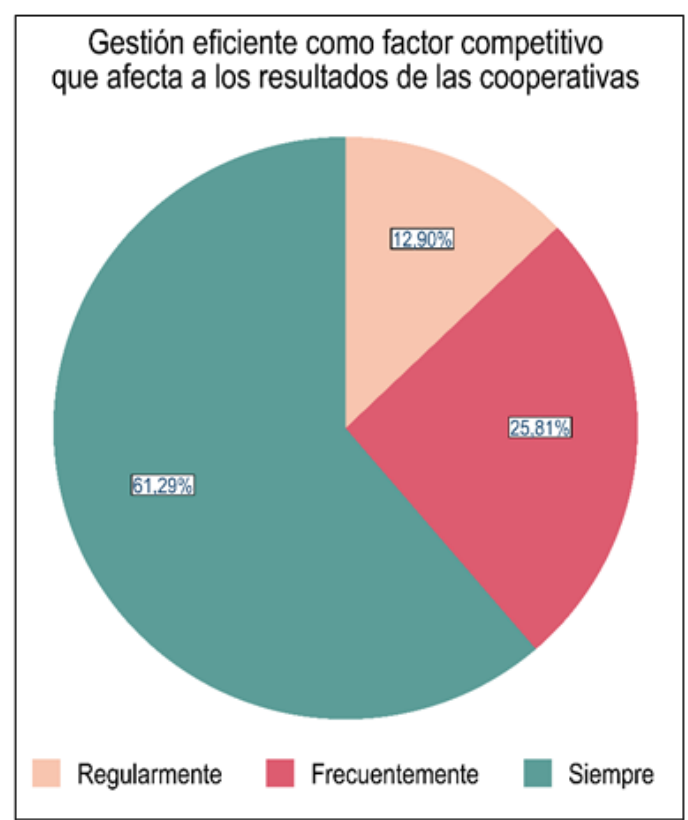

Figura 2. Gestión financiera eficiente.

\section{Fuente: Los Autores}

Si se considera la gestión financiera desde el punto de vista de la eficiencia para tomarla como factor competitivo que impacte en los resultados de las cooperativas, se parecía que el $61,29 \%$ de la muestra encuestada, valor equivalente a 19 entidades de las 31 siempre toman este factor de esta manera. Un $25,81 \%$ equivalente a 8 entidades 


\section{LA GESTIÓN FINANCIERA COMO FACTOR DE LA RENTABILIDAD EN LAS COOPERATIVAS DE AHORRO Y CRÉDITO DEL ECUADOR 2016-2020}

toman la gestión financiera frecuentemente como elemento de eficiencia que impacto en los resultados y un $12,9 \%$ ( 4 entidades) regularmente lo hacen. Esta distribución indica que la gestión financiera eficiente es un elemento constante en todas las entidades y que necesariamente será considerado en la ponderación de los resultados para una mejora de estos. A diferencia del anterior indicador, donde la gestión financiera se veía como un factor competitivo para la generación de rentabilidad, éste cuenta con un alto grado de afinidad y aprobación a nivel de todas las entidades, por lo mismo que ninguna de las encuestas declara que Nunca se considera de esta manera al indicador.

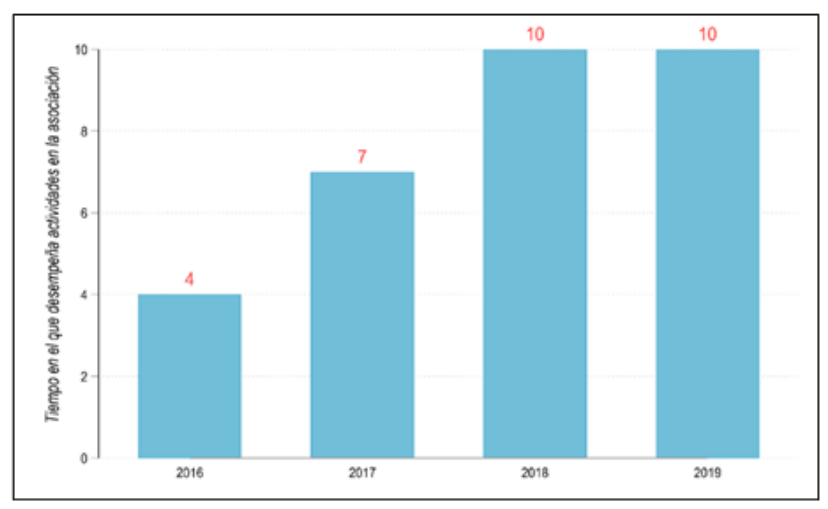

Figura 3. Tiempo.

\section{Fuente: Los Autores}

En cuanto al tiempo en el que se desarrollan las actividades dentro de las entidades, se aprecia que el tiempo promedio a nivel de las entidades ha ido creciendo progresivamente y se ha estancado en 10 años desde el 2018 y 2019. Dicha proporción de tiempo representa un total del $32,26 \%$ de los encuestados mientras que el tiempo más bajo es evidentemente el del 2016, primer año donde se registra información con un tiempo de 4 años representando un total de $12,90 \%$ de la muestra.

La renovación de los productos ofrecidos por las entidades es un mecanismo para poder seguir generando nuevos ingresos y afianzamiento de los clientes. Por ello es importante generar nuevos incentivos en el servicio ofrecido para poder tener una dinámica estable en el mercado. En ese sentido, un $93,55 \%$ de la muestra encuestada equivalente a 29 entidades considera que su cooperativa SI ha considerado elaborar productos nuevos para incrementar la rentabilidad. Por otro lado, un $6,45 \%$ equivalente a 2 entidades consideran REGULARMENTE la generación de nuevos productos. La capacitación de los recursos humanos en toda entidad juega un factor sumamente importante y ayuda a generar el valor agregado de la misma en la medida que un factor humano efectiva y eficientemente capacitado afianza su vínculo a la empresa y usa los nuevos conocimientos para mejorar el desarrollo de actividades en la empresa. Por ello, un 19,35\% de las entidades registradas en la muestra, equivalente a 6 , consideran que su entidad SIEMPRE considera el recurso humano ha sido capacitado de acuerdo a su competencia desarrollada para mejorar sus resultados. Un 16,13\% (5 entidades) consideran dicha condición como FRECUENTEMENTE, un 16,13\% (5 entidades) también considera esta condición ESPORÁDICAMENTE, y finalmente un sólido 49,39\% (15 entidades) consideran que chica condición se da REGULARMENTE en su entidad.

La lógica de la oferta y la demanda establecen que la mejora medida de conseguir una mayor rentabilidad es reduciendo los costos implícitos en la generación de un bien o servicio ofrecido. Por ello, la reducción de costos de operación podría implicar que, si en caso se redujeran, habría la posibilidad de que la rentabilidad de la entidad se incremente. Así, el $54,84 \%$ de las entidades (27 de estas) creen que esto sucede SIEMPRE en su cooperativa, y un 12,90\% (4 entidades) declara que FRECUENTEMENTE se da dicha situación.

El apalancamiento es la capacidad de una entidad para poder formar capital adicional

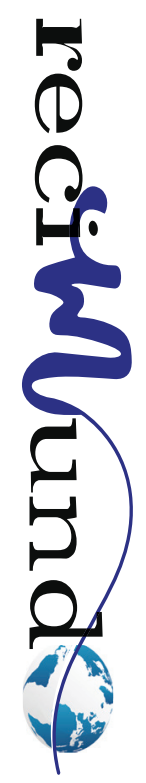


a base de un préstamo. Mientras mayor sea la capacidad de apalancamiento, esto es sinónimo de buena estabilidad financiera y gran cumplimiento y récord crediticio de la misma. En ese sentido, un 80,65\% (25 entidades) de los reportes de la muestra consideran que su cooperativa SI considera que, si su entidad obtiene financiamientos externos, tendría mayor oportunidad para crecer en el mercado y por ende la rentabilidad. Mientras que un 19,35\% (6 entidades) reportan que esto se considera REGULAR (MEDIANAMENTE).

En cuanto al comportamiento de las ventas que realiza la cooperativa, se registra que todas perciben como BUENO el comportamiento de las ventas registradas.

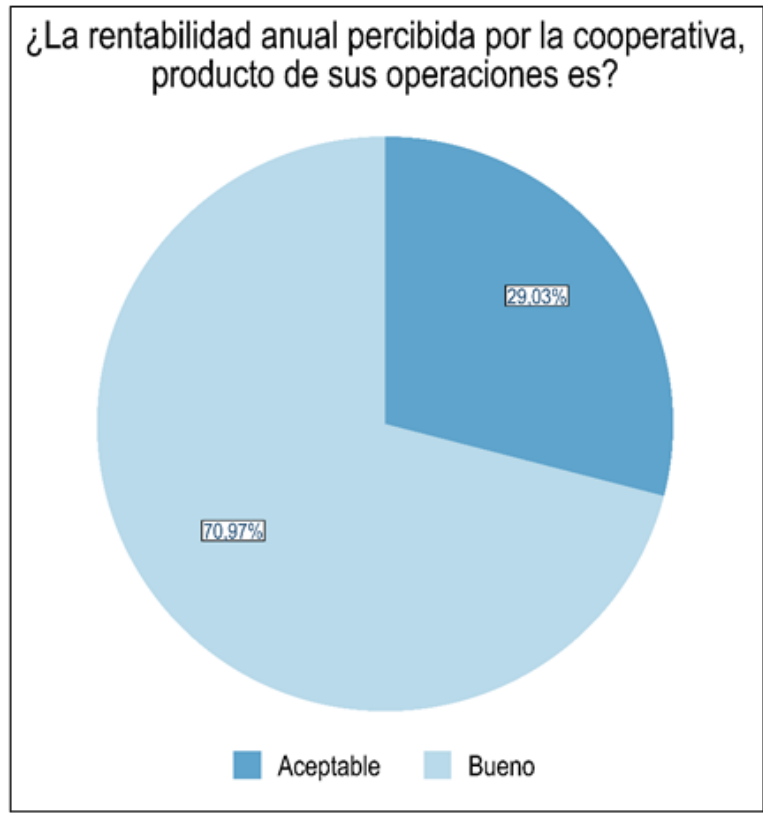

Figura 4. Utilidad Operacional.

\section{Fuente: Los Autores}

La rentabilidad anual de la cooperativa es uno de los elementos primordiales al ver que tan viable es continuar la operación de esta. Por ello los datos que se registran a nivel de los encuestados reportan que un $70,97 \%$ (22 encuestados) creen que dicho evento es ACEPTABLE, un 29,03\% (9 encuestados) creen que esto es BUENO.

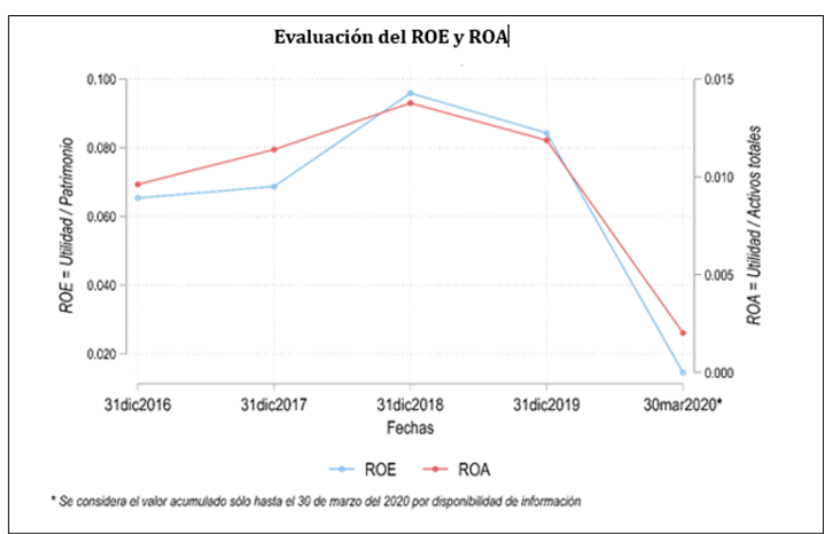

Figura 5. $R O A$ - ROE 2016-2020

Fuente: Los Autores

A primera vista se puede apreciar que tanto el ROE como el ROA tienen un comportamiento claramente sincrónico y en los primeros períodos se aprecia una tendencia evidentemente creciente en ambos casos. Sin embargo, entre 2018 y 2019 se aprecia que dicha tendencia sufre un evidente quiebre posiblemente debido al contexto general de caída de los índices internacionales. Por ello, estos índices reportan una ligera caída en la tendencia que mantenían por 2 períodos consecutivos. En cuanto al efecto registrado en 2020, es importante recalcar que solamente se cuenta con valores hasta el mes de marzo del año en mención, por lo que evidentemente existe una brecha de valores que no se llegaron a reportar dado que es la única información disponible a este nivel. 


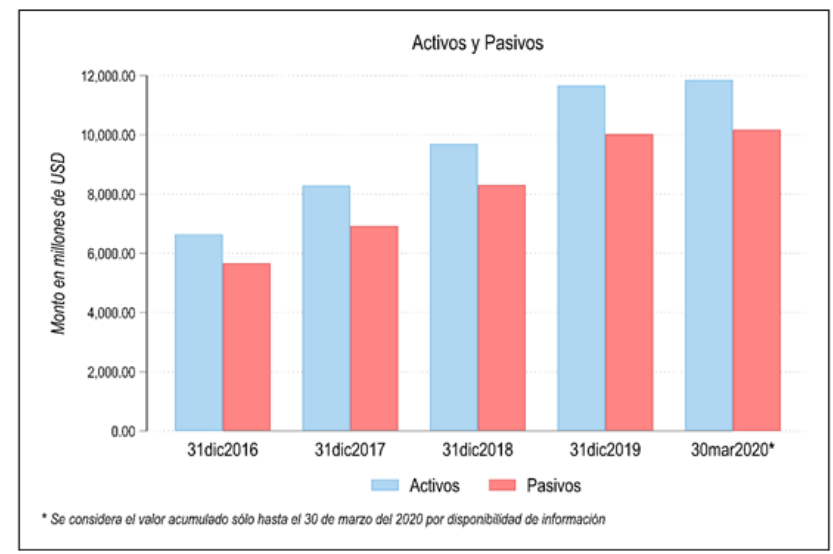

Figura 6. Activos y Pasivos.

Fuente: Los Autores

En cuanto al valor registrado a nivel de Activos y Pasivos se puede apreciar que ambos han ido creciendo a un ritmo constantes desde el 2016 al 2020 y las diferencias entre el valor registrado entre ambos se mantiene y en cierta medida se aprecia una reducción de dicha brecha de manera "rápida", la cual en promedio ascendió a $15.92 \%$ en el caso los Activos y en promedio a $16.08 \%$ para los Pasivos, considerando todos los períodos de estudio. La diferencia entre ambos valores en promedio para todos los periodos considerados dentro de la presente investigación versa en torno a USD 1,414.22 millones, registrando su menor valor para el año 2016 con USD 977.09 millones y la mayor en el último período con USD 1682.45 millones. Cabe considerar que este crecimiento sostenido en ambos valores responde a un comportamiento bastante estable de las entidades y un criterio de estabilidad a pesar de que durante los años intermedios de estudio los niveles de endeudamiento a nivel general estuvieron perdiendo su nivel promedio a 10 años.

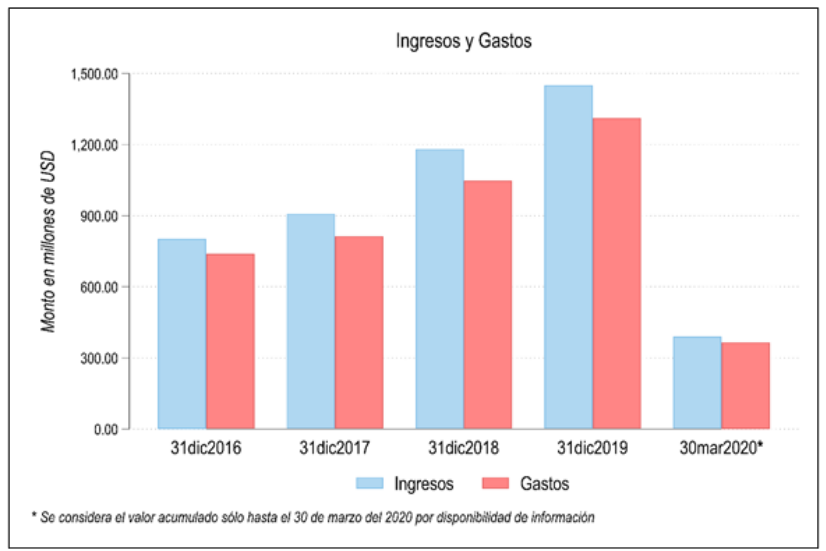

Figura 7. Ingresos y gastos.

Fuente: Los Autores

En cuanto a los ingresos y gastos se aprecia que existe una tendencia creciente que se mantiene en los diferentes períodos y nuevamente cabe mencionar que el valor registrado a nivel del año 2020 sólo considera los 3 primeros meses de ese año, a diferencia del resto de montos que acumulan el valor de 12 meses correspondientes a cada uno de los años de estudio. A partir del gráfico se puede apreciar un crecimiento constante, el cual en promedio se ubica en $21,9 \%$ para el caso de los ingresos y en $21,36 \%$ para los gastos, en ambos casos sin considerar la tasa de 2020 puesto que no presenta un valor comparable con el resto de los montos acumulados. Por otro lado, se aprecia que, en el período del 31 de diciembre de 2018, la tasa de crecimiento tanto para ingresos como para gastos fue la mayor de todos los registros, posicionándose en un valor de 30,4\% para el caso de ingreso y $28,7 \%$ para gastos. Sin embargo, este comportamiento, a pesar de mantener la tendencia creciente, sufre un ligero retroceso en su intensidad pasando a reportar únicamente tasas de $22,83 \%$ y $25,26 \%$ para ingresos y gastos respectivamente en el año 2019. En cuanto a las diferencias registradas entre los montos por año, se aprecia que en promedio la diferencia promedio entre todos los períodos reporta un valor de USD 107,56 millones y la más alta se reporta en el registro del año 2019 con USD 138,46 millones. 
En cuanto a la información del Patrimonio de las entidades, se aprecia que existe una tasa de crecimiento constante, pero que presenta una ligera pausa entre el cambio de 2017 a 2018, registrando únicamente un crecimiento de $1,22 \%$ respecto al año anterior. Esto nuevamente puede deberse a una eventualidad coyuntural asociada a las fluctuaciones que se dio en dicho periodo en el entorno económico y político nacional. A pesar de ello, el promedio de la tasa de crecimiento del patrimonio considerando el valor hasta finales de 2019 se ubica en una tasa de 20,02\% y el período donde se registra el mayor crecimiento entre todos es el del año 2017, con una tasa de 40,79\% respecto al año anterior. En cuanto a la información registrada para 2020, se parecía que dicha tasa de crecimiento mantiene su tendencia, considerando que el monto solamente cuenta con una acumulación de los primeros 3 meses mientras que el resto de información de los períodos anteriores cuenta con una acumulación de los 12 meses correspondientes a todos los años reportados en la presente investigación.

\section{Análisis a partir de los indicadores}

Del total de las Cooperativas de Ahorro y Crédito (CAC), 4 de ellas que representan el $12.90 \%$ muestran preferencia por la categoría REGULAR en cuanto a la que la Gestión Financiera es un factor competitivo, mientras que el resto de las 27 consideran que el comportamiento es ACEPTABLE.

Del total de las CAC, 4 de ellas que representan el $12.9 \%$ muestran preferencia por la categoría frecuentemente en cuanto a que el desempeño financiero es un factor competitivo, 8 de ellas que representan el $25.8 \%$ por la categoría nunca, 7 de ellas que presentan el $22.6 \%$ por la categoría siempre y 6 de ellas que representan el 19.4\% señalan que regularmente el desempeño financiero es un factor competitivo.
Del total de las CAC, 22 de ellas que representan el $70.97 \%$ muestran una valoración por la categoría REGULAR en cuanto a la rentabilidad y solamente 9 de ellas que representan el 29.03\% reflejan una valoración REGULAR en relación con la rentabilidad.

Dado que las respuestas registradas para el indicador, a partir de la consolidación de los componentes principales resulta que no existe varianza para el resultado, solo se tiene que el $100 \%$ de la muestra considera los ingresos netos bajo la categoría de REGULAR.

Del total de las CAC encuestadas, 22 de ellas que representan el $70.97 \%$ muestran una valoración por la categoría REGULAR en cuanto a la utilidad operacional, y solo 9 de ellas que representan el $29.03 \%$ reflejan una valoración ACEPTABLE en relación a la utilidad operacional.

Prueba de Hipótesis: Contraste de hipótesis Hipótesis General.

HO: La gestión financiera influye en la rentabilidad de las Cooperativas de Ahorro y Crédito de la Economía Popular y Solidaria del Ecuador, 2016- 2020.

H1: La gestión financiera Sí influye en la rentabilidad de las Cooperativas de Ahorro y Crédito de la Economía Popular y Solidaria del Ecuador, 2016- 2020. 


\section{LA GESTIÓN FINANCIERA COMO FACTOR DE LA RENTABILIDAD EN LAS COOPERATIVAS DE AHORRO Y CRÉDITO DEL ECUADOR 2016-2020}

Tabla 4. Correlación de Spearman para Gestión Financiera y la Rentabilidad.

\begin{tabular}{|c|c|c|c|c|}
\hline \multicolumn{5}{|c|}{ Correlaciones } \\
\hline & & & $\begin{array}{l}\text { Gestión } \\
\text { Financiera }\end{array}$ & Rentabilidad \\
\hline \multirow[t]{6}{*}{ Rho de Spearman } & Gestión Financiera & Coeficiente de correlación & 1,000 & .631 \\
\hline & & Sig. (bilateral) & &, 000 \\
\hline & & $\mathrm{N}$ & 31 & 31 \\
\hline & Rentabilidad & Coeficiente de correlación &, 631 & 1,000 \\
\hline & & Sig. (bilateral) &, 000 & . \\
\hline & & $\mathrm{N}$ & 31 & 31 \\
\hline
\end{tabular}

\section{Fuente: Los Autores}

El coeficiente de correlación 0,631, indica que existe una correlación moderada, directa y positiva entre las variables, con un p-valor de 0,00 que resulta menor al valor 0,05 de la significancia. Por tanto, se tiene evidencia estadística para afirmar que: La gestión financiera Sí influye en la rentabilidad de las Cooperativas de Ahorro y Crédito de la Economía Popular y Solidaria del Ecuador, 2016- 2020.

\section{Hipótesis Específica}

HO: El desempeño financiero incide en la rentabilidad de las Cooperativas de Ahorro y Crédito de la Economía Popular y Solidaria del Ecuador, en el periodo 2016-2020.

H1: El desempeño financiero, Sí incide en la rentabilidad de las Cooperativas de Ahorro y Crédito de la Economía Popular y Solidaria del Ecuador, en el periodo 2016-2020.

Tabla 5. Correlación de Spearman para el Desempeño Financiero y la Rentabilidad.

\begin{tabular}{|c|c|c|c|c|}
\hline \multicolumn{5}{|c|}{ Correlaciones } \\
\hline & & & Desempeño & Rentabilidad \\
\hline \multirow[t]{6}{*}{ Rho de Spearman } & Desempeño & Coeficiente de correlación & 1,000 & 419 \\
\hline & & Sig. (bilateral) & . &, 019 \\
\hline & & $\mathrm{N}$ & 31 & 31 \\
\hline & Rentabilidad & Coeficiente de correlación & 419 & 1,000 \\
\hline & & Sig. (bilateral) &, 019 & . \\
\hline & & $\mathrm{N}$ & 31 & 31 \\
\hline
\end{tabular}

Fuente: Los Autores

El valor del coeficiente de correlación rho es de 0,4192, e indica que existe una correlación medianamente baja, directa y positiva entre las variables, con un p-valor de 0,019 que resulta menor al valor 0,05 de la significancia establecida en los criterios de la evaluación. Por tanto, se afirma que: El desempeño financiero, Sí incide en la ren- tabilidad de las Cooperativas de Ahorro y Crédito de la Economía Popular y Solidaria del Ecuador, en el periodo 2016-2020

\section{Discusión de Resultados}

Esta investigación tuvo como objetivo determinar si la gestión financiera influye en 
la rentabilidad del Sector Asociativo de las Cooperativas de Ahorro y Crédito de la Economía Popular y Solidaria del Ecuador 2016-2020.

Los resultados obtenidos confirman la hipótesis general, manifestando que la variable Gestión Financiera SI influye de manera positiva con la rentabilidad de las Cooperativas de Ahorro y Crédito de la Economía Popular y Solidaria del Ecuador, 2016- 2020.

Según estudio realizado por la Cooperativa de las Américas, Región de la Alianza de las cooperativas Internacional, 2014: Director Regional Manuel Mariño: establece: que la gestión financiera es el resultado de la acción conjunta de varios elementos complementarios: i) entorno regulatorio propicio; ii) oferta de productos adecuados (pagos, ahorros, créditos, seguros); iii) cobertura, con acceso a canales transaccionales tradicionales y no tradicionales para recibir servicios de calidad a precios razonables, que permitan realizar las operaciones en forma segura y eficiente, iv) fomento y difusión de la educación, alfabetización y cultura financieras; y v) protección al usuario de los servicios y transparencia de la información.

En lo que se refiere a las Hipótesis secundarias, se detallarán a continuación las siguientes conclusiones: Se obtuvo como resultado que la innovación $\mathrm{SI}$ influye en la rentabilidad de las Cooperativas de Ahorro y Crédito de la Economía Popular y Solidaria del Ecuador, 2016- 2020.

Se obtuvo como resultado que el desempeño financiero SI incide en la rentabilidad de las Cooperativas de Ahorro y Crédito de la Economía Popular y Solidaria del Ecuador, 2016- 2020.

Así también se determinó que la calidad del servicio SI incide en la rentabilidad de las Cooperativas de Ahorro y Crédito de la Economía Popular y Solidaria del Ecuador, 2016- 2020, sustentado en que las Coope- rativas de Ahorro y Crédito poseen energía, capacidad de riesgo, perseverancia y creatividad para hacer frente a los retos y mejorar la calidad de servicio a sus socios, tal como lo estamos viviendo en la actualidad en la época post pandemia.

\section{Conclusiones}

- La gestión financiera Sí influye en la rentabilidad de las Cooperativas de Ahorro y Crédito de la Economía Popular y Solidaria del Ecuador, 2016- 2020.

- Las Cooperativas de Ahorro y Crédito de la Economía Popular y Solidaria del Ecuador que innovan, optimizando sus procesos operativos de gestión financiera, Sí, obtienen mejor rentabilidad en los resultados del periodo 2016-2020.

- El desempeño financiero, Sí incide en la rentabilidad de las Cooperativas de Ahorro y Crédito de la Economía Popular y Solidaria del Ecuador, en el periodo 2016-2020.

- La calidad del servicio, Sí incide en la rentabilidad de las Cooperativas de Ahorro y Crédito de la Economía Popular y Solidaria del Ecuador, en el periodo 2016- 2020.

- Se determinó que la rentabilidad sobre patrimonio y sobre los activos el ROE y ROA, de las Cooperativas de Ahorro y Crédito de la Economía Popular y Solidaria del Ecuador, 2016- 2020, segmento 1 , presentan en los primeros períodos de estudio una tendencia creciente en ambos caso, 2016-2019, sin embargo, entre 2019 y 2020 se aprecia una disminución debido al contexto económico interno del país, en tanto que el año 2020, la crisis económica profundizada por la pandemia provocó que el sector cooperativo de ahorro y crédito del país no alcance los rendimientos del 2019, así presenta:

ROE: $2016=0.07 ; 2017=0.07 ; 2018=0.10$; $2019=0.08 ; 2020($ marzo $)=0.01$ 


\section{LA GESTIÓN FINANCIERA COMO FACTOR DE LA RENTABILIDAD EN LAS COOPERATIVAS DE AHORRO Y CRÉDITO DEL ECUADOR 2016-2020}

ROA: $2016=0.01 ; 2017=0.01 ; 2018=$ $0.01 ; 2019=0.01 ; 2020($ marzo $)=0.00$

\section{Bibliografía}

Asamblea Nacional. (2008). Constitucion de la Republica del Ecuador. 139. Quito, Ecuador: Corporacion de estudios y publicaciones.

Asamblea Nacional. (2008). Ley de Economía Popular y Solidaria en Ecuador. Quito.: Constitución de 2008.

Asamblea Nacional. (2015). Ley de Economía Popular y Solidaria en Ecuador. Quito.: Constitución de 2008.

Barillas, M. A. (2012). Foro Económico Mundial sobre America Latina Transformación Regional en un Nuevo Contexto Global. Puerto Vallarta, México.

Bojórquez, M. I., Perez, A. E., \& Gonzalez, B. M. (2013). La gestion financiera como factor de competitividad para las Pymes familiares en la industrial textil, en Yucatan, México. Global conferencia on bussines and finance proceedings, 8 .

Burk, J., \& Lehnman, R. (2004). Financing Your Small Business. Sphinx Publishing.

Córdoba, P. M. (2013). Gestion financiera. Bogotá: Ecoe Ediciones.

Correa, J. A., Ramírez, B. L., \& Castaño, R. C. (2009). Modelo de gestión financiera integral para MIPYMES en Colombia. 1.

Delfín, P. F., \& Acosta, M. M. (2016). Importancia y análisis del desarrollo empresarial. Pensamiento \& Gestion, Barranquilla Colombia.

Fernández, I. (2005). http://uptparia.edu.ve/documentos/F\%C3\%ADsico\%20de\%20Escala\%20Likert.pd.

Franklin, E. B. (2007). Auditoria Administrativa, Gestion Estrategica del Cambio. México: PEARSON EDUCACION.
Germán Guerrero Chaparro. (JULIO-DICIEMBRE de 2003). Los retos de la gestión financiera frente a la planeación estratégica de las organizaciones y la globalización. (G. G. Chaparro*, Ed.) INNOVAR, revista de ciencias administrativas y sociales. No. 21(21), 46.

Jordan, R. W. (2006). Fundamentos de Finanzas Corporativas. (7 a Edición. ed.). (J. M. Chacón., Ed.) México: McGraw.Hill interamericana.

José Luis Coraggio. (2007). La Economia Popular y Solidaria en el Ecuador.

Luque González, A., \& Peñaherrera Melo, J. (2021). Cooperativas de ahorro y crédito en Ecuador: el desafío de ser cooperativas. Revesco, 138, 1-17.

Montenegro, J. L. (2013). El calentamiento financiero global. México.

Nacional, A. (2008). Ley de Economía Popular y Solidaria en Ecuador. Quito.: Constitución de 2008.

Nevado, D. P. (2006). Cómo Gestionar el Binomio Rentabilidd Productividad. Madrid: Especial Directivos.

Ortìz Gonzàlez, J. (09 de 01 de 2015). Obtenido de http://finanzasyproyectos.net/como-evaluar-la-gestion-financiera/

Pozos, F. L., \& Márquez, M. (2016). Importancia y análisis del desarrollo empresarial. Pensamiento \& gestión, (40),, 40, 184-202. doi: http://dx.doi. org/10.14482/pege.40.8810

Red Financiera Rural. (s.f.). Microfinanzas Ecuador. Microfinanzas.

Rodríguez. (febrero de 2014). La gestión económico-financiera bajo los modelos de colaboración público privada. T35595. Madrid, España: UNIVERSIDAD COMPLUTENSE DE MADRID,FACULTAD DE CIENCIAS ECONÓMICAS Y EMPRESARIALES, TESIS DOCTORAL.

SEPS. (2017). https://www.seps.gob.ec/documents/20181/594508/NOTA+TE\%CC\%81C$\mathrm{NICA}+\mathrm{PARA}+\mathrm{P} U \mathrm{BLICAR}+-\mathrm{FICHA}+\mathrm{ME}-$ TODOLOGICAS+DE+INDICADORES.pdf/ a71e5ed1-7fae-4013-a78d-425243db4cfa.

\section{CITAR ESTE ARTICULO:}

Yaguache Maza, D. M., \& Hennings Otoya, J. A. (2021). La gestión financiera como factor de la rentabilidad en las cooperativas de ahorro y crédito del Ecuador 2016-2020. RECIMUNDO, 5(4), 356-371. https://doi.org/10.26820/recimundo/5.(4).dic.2021.356-371

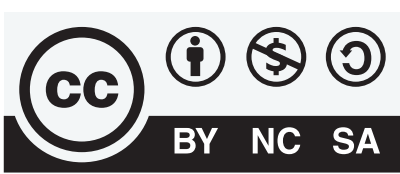

CREATIVE COMMONS RECONOCIMIENTO-NOCOMERCIAL-COMPARTIRIGUAL 4.0.

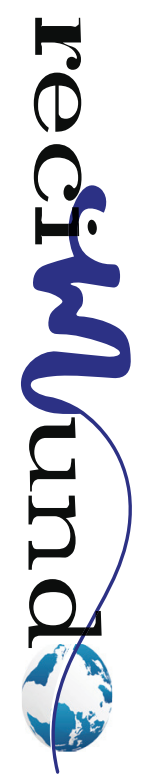

